\title{
Anlotinib induces tumor blood vessel normalization to strengthen the anticancer effect of radiotherapy on esophageal cancer by inhibiting EphA2
}

Weiguo Zhu ( $D$ Zhuweigu02233@163.com )

the affiliated huainan No.1 people's hospital of nanjing medical university

Jing Huang

the affiliated huaian no.1 people's hospital of nanjing medical university

Zhenlin Gu

the affiliated huaian no.1 people's hospital of nanjing medical university

Jiasheng Huang

the affiliated huaian no.1 people's hospital of nanjing medical university

Yingying $\mathrm{Xu}$

the affiliated huaian no.1 people's hospital of nanjing medical university

Wanwei Wang

the affiliated huaian no.1 people's hospital of nanjing medical university

\section{Nano Express}

Keywords: Esophageal cancer, Anlotinib, Ephrin type-A receptor 2, Radioresistance, Angiogenesis, Proliferation, Invasion, Migration

Posted Date: July 13th, 2021

DOl: https://doi.org/10.21203/rs.3.rs-681721/v1

License: (9) This work is licensed under a Creative Commons Attribution 4.0 International License. Read Full License 


\section{Abstract \\ Background}

Anlotinib has anti-tumor activity in diverse solid tumors. Given that, our study was designed to unearth the mechanism of Anlotinib in radioresistant esophageal cancer (EC) cells by modulating Ephrin type-A receptor 2 (EphA2).

\section{Methods}

EC cells (TE-1 and KYSE-150) were induced to radioresistant EC cells (TE-1R and KYSE-150R). EphA2 expression in TE-1R and KYSE-150R cells was measured. Then, TE-1R and KYSE-150R cells were treated with Anlotinib and/or transfected with the plasmids that altered EphA2 expression. Otherwise, TE-1R and KYSE-150R cells were transfected with si-EphA2 or OE-EphA2 plasmids independently. In vitro experiments were conducted to monitor cell proliferation, angiogenesis, migration and invasion. In vivo experiment was also implemented to observe tumor growth.

\section{Results}

EphA2 expression was raised in TE-1R and KYSE-150R cells. Anlotinib inhibited proliferation, angiogenesis, migration and invasion of TE-1R and KYSE-150R cells in vitro, as well as tumor growth and MVD in vivo. Inhibiting EphA2 enhanced Anlotinib-mediated effects on TE-1R and KYSE-150R cells. Down-regulating EphA2 restrained proliferation, angiogenesis, migration and invasion of TE-1R and KYSE-150R cells.

\section{Conclusion}

It is concluded that Anlotinib suppresses EC development under radiotherapy by inhibiting EphA2, providing another perspective to overcome radioresistance in EC.

\section{Background}

As a heterogeneous malignancy, esophageal cancer (EC) is mostly diagnosed in advanced stages and esophageal squamous cell carcinoma (ESCC) accounts for most cases of the disease [1]. Demographics, smoking, alcohol consumption, gastroesophageal reflux disease, obesity and diet are commonly considered as the risk factors for EC [2]. EC is usually asymptomatic in the early stages and advanced disease may accompany heartburn unresponsive to medication, unconscious weight loss, progressive dysphagia, signs of blood loss, chest pain or odynophagia [3]. Multimodality approaches such as endoscopic mucosal resection and endoscopic submucosal dissection, surgical treatment, neoadjuvant and adjuvant chemotherapy as well as concurrent chemoradiotherapy have been developed in the 
treatment of EC [4]. However, tumor-associated microenvironmental factors and cellular mechanisms may lead to radioresistance [5]. Thus, dealing with radioresistance may be a practical approach to manage EC. Blood vessel normalization in tumors could reduce tumor uptake and intratumoral accumulation [6] and tumor blood vessel normalization and ES radiotherapy need further research.

Anlotinib is an orally administered tyrosine kinase inhibitor that is designed to inhibit angiogenesis and growth of tumors [7]. More importantly, Anlotinib could reduce blood vessel sprout and microvessel density (MVD), and restrain migration and tube formation in tumors [8]. In fact, Anlotinib has great therapeutic efficacy in treating cancers, such as advanced non-small cell lung cancer (NSCLC), advanced soft tissue sarcoma and metastatic renal cell carcinoma [9]. In ESCC, it has been reported that Antutinib combined with radiotherapy and chemotherapy has strong anti-tumor effects on patient-derived xenografts bearing mice [10]. In a clinical trail, it has been found that Antutinib combined with chemotherapy could improve the survival of patients with advanced ESCC [11], while Antutinib's treatment mechanism for ES still needs further study. Ephrin type-A receptor 2 (EphA2) is a tyrosine kinase that is richly produced in tumors and regulation of EphA2 confers a potential in managing tumors [12]. EphA2 is found to act as a tumor-associated surface antigen of chimeric antigen receptor in the case of ESCC treatment [13]. It has been further analyzed that regulating EphA2 expression is a mediator for vasculogenic mimicry of EC cells [14]. In ESCC samples after radiotherapy, genomic profile of EphA2 is altered and absence of mutation of EphA2 confers to radioresistance [15]. In endometrial cancer, EphA2 overexpression is positively correlated with high VEGF expression, which is associated with angiogenesis and disease-specific survival of patients [16]. Referring to these reports, we assumed that Anlotinib inhibits EphA2 to induce normalization of tumor blood vessels and suppress biological functions of radioresistant $\mathrm{EC}$ cells, and may renew the mechanism underlying radioresistance of $\mathrm{EC}$ and provide the therapeutic reference for control EC development.

\section{Methods And Materials \\ 2.1 Ethics statement}

Animal experiments have been reviewed and approved by the animal ethics committee of The Affiliated Huaian No.1 People's Hospital of Nanjing Medical University.

\subsection{Cell culture}

Human normal esophageal epithelial cells (THEECs) and EC cell lines TE-1 and KYSE-150 (ATCC, VA, USA) were kept in Roswell Park Memorial Institute (RPMI)-1640 (10\% fetal bovine serum [FBS], 100 unit/mL penicillin and $100 \mathrm{mg} / \mathrm{mL}$ streptomycin). The medium were all provided by Gibco (NY, USA).

\subsection{Induction of radioresistance in EC cells}

Radioresistant EC cell lines (TE-1R and KYSE-150R) were induced through multiple fractionated irradiation [17]. TE-1 and KYSE-150 cells $\left(1.5 \times 10^{6} \mathrm{cells}\right)$ in a culture flask $\left(25 \mathrm{~cm}^{2}\right)$ were irradiated with 1 Gy X-ray and immediately transferred into a fresh medium. When cells reached $90 \%$ confluence, they were 
cultured in a new culture flask and treated with a second irradiation at $50 \%$ confluence. Totally, cells were irradiated at 1 Gy three times, 2 Gy three times, and 4 Gy three times.

\subsection{Colony formation assay}

Colony formation assay was utilized to assess the radioresistance of parental and resistant EC cells. Parental and resistant EC cells in the log phase were trypsinized and seeded into 100-mm petri dishes. Upon cell adherence, cells were irradiated with 0, 2, 4, 6, 8 and 10 Gy X-ray, respectively and continuously cultured for 12 days to observe cell colonies.

\subsection{Cell transfection}

Cells in the log phase were cultured on a 6-well plate containing RPMI-1640 (2 × $10^{5}$ cells/well). Cells at 90\% confluence were transfected with EphA2-negative control (CTRL), siRNA-EphA2 or overexpression (OE)-EphA2 (GenePharma, Shanghai, China) via Lipofection ${ }^{\text {TM }}$ (InivoGene, CA, USA). Three replicate wells were set.

\subsection{Anlotinib treatment}

Cells were treated with Anlotinib (CTTQ, Jiangsu, China) at 2, 4 and $8 \mu \mathrm{mol} / \mathrm{L}$, respectively for $48 \mathrm{~h}$. A control was established with cells treated with saline [18].

\subsection{3-(4, 5-dimethylthiazol-2-yl)-2, 5-diphenyltetrazolium bromide (MTT) assay}

Cells were placed in a 96-well plate at $3 \times 10^{3}$ cells/well. After $48 \mathrm{~h}$, cells were combined with MTT solution at $20 \mu \mathrm{L} /$ well (Beyotime, Shanghai, China) for $4 \mathrm{~h}$, and with Dimethyl Sulfoxide at $100 \mu \mathrm{L} / \mathrm{well}$. D value at $490 \mathrm{~nm}$ was recorded on an automatic microplate reader (Tecan M200, TECAN, Switzerland).

\subsection{Tube formation assay}

Cells were cultured in a serum-free medium for $24 \mathrm{~h}$ and then in a medium containing $10 \%$ FBS. Then, the supernatant was centrifuged at $1000 \mathrm{r} / \mathrm{min}$ and filtered through a filter $(0.22 \mu \mathrm{m})$ to obtain the conditioned medium (CM), which was preserved at $4^{\circ} \mathrm{C}$. A mixture $(40 \mu \mathrm{L})$ made by the $\mathrm{CM}$ and Matrigel (1:1) was spread on a 96-well plate overnight and incubated with human umbilical vein endothelial cell suspension $\left(1 \times 10^{5}\right.$ cells $\left./ \mathrm{mL}\right)$ at $200 \mu \mathrm{L} /$ well. The formed tubes were observed and counted in 4 fields of view by a microscope (Olympus, Tokyo, Japan) [18].

\subsection{Transwell assay}

Cells were prepared into a single cell suspension with serum-free Dulbecco's Modified Eagle Medium (DMEM). The cell suspension $\left(100 \mu \mathrm{L}, 3 \times 10^{5}\right.$ cells $\left./ \mathrm{mL}\right)$ was added to the upper chamber of Transwell (Corning, N.Y., USA). Matrigel (BD Company, NJ, USA) was used for invasion assay but not for migration assay. The bottom chamber was supplemented with 10\% FBS-DMEM (600 $\mu \mathrm{L})$. After $24 \mathrm{~h}$, cells were fixed with $95 \%$ ethanol, stained with crystal violet and counted under a microscope. 


\subsection{Tumor xenografts in nude mice}

Male and female BALB/c(nu/nu) nude mice (4-6 weeks old; $15-18 \mathrm{~g}$ ) were provided by Beijing Vital River Laboratory Animal Technology Co., Ltd. (Beijing, China). Mice were housed in specific pathogenfree-level animal barrier $\left(18-23{ }^{\circ} \mathrm{C}\right.$, humidity $50-60 \%, 12 \mathrm{~h}$ day/night alternate, disinfected food and water). A week later, the skin of the left back of mice was sterilized with ethanol, and mice were subcutaneously injected with $100 \mu \mathrm{L}$ of cell suspension $\left(1 \times 10^{6}\right.$ cells $\left./ \mathrm{mL}\right)$ into the back. In the following 2 weeks, general condition of the mice and local condition of injection site were observed. Mice were divided into three groups: KYSE-150R group, KYSE-150R + X-ray group, and KYSE-150R + Anlotinib + X-ray group. Mice in the KYSE-150R + Anlotinib + X-ray group were given $1.5 \mathrm{mg} / \mathrm{kg}$ Anlotinib by intragastric administration for 2 weeks. Mice in the KYSE-150R + X-ray group and KYSE-150R + Anlotinib + X-ray group were irradiated with $6 \mathrm{~Gy}$ X-ray every week. At 4 weeks post injection, mice were euthanized, the excised tumors were weighed, and tumor volume was measured [18].

\subsection{Reverse transcription quantitative polymerase chain reaction (RT-qPCR)}

After extraction of total RNA in tissues and cells by Trizol (Invitrogen, CA, USA), RNA concentration was determined with Nanodrop 2000 (Thermo Fisher Scientific, MA, USA) and reverse transcribed to cDNA using PrimeScript RT kit (Takara, Kyoto, Japan). Using SYBR Premix Ex Taq kit (Tli RNaseH Plus) kit (Takara), real-time PCR was performed on an ABI7500 (Thermo Fisher Scientific). EphA2 expression was calculated by $2^{-\triangle \triangle \mathrm{Ct}}$ method and normalized to glyceraldehyde-3-phosphate dehydrogenase (GAPDH). The primers (GenePharma) were shown in Table 1.

Table 1

Primer sequences

\begin{tabular}{|ll|}
\hline Genes & Primers $\left(\mathbf{5}^{\left.\boldsymbol{} \rightarrow \mathbf{3}^{\prime}\right)}\right.$ \\
\hline EphA2 & F: ACTGCCAGTGTCAGCATCAACC \\
\hline R: GTGACCTCGTACTTCCACACTC \\
\hline GAPDH & R: GCTCTCTGCTCCTCCTGTTC \\
\hline Note: EphA2, Ephrin type-A receptor 2; GAPDH, glyceraldehyde-3-phosphate dehydrogenase \\
\hline
\end{tabular}

\subsection{Western blot assay}

After extraction of protein in tissues and cells, protein concentration was measured by bicinchoninic acid method. The protein was mixed with loading buffer at 2:1 and denatured. After separation by sodium dodecyl sulfate-polyacrylamide gel electrophoresis, protein was transferred to a polyvinylidene fluoride membrane and combined with primary antibodies EphA2 (1:2000, Thermo Fisher Scientific), VEGF (1:2000, Abcam), basic fibroblast growth factor (bFGF; 1:1000, Abcam), and GAPDH (1:1000, Millipore, 
MA, USA). Afterwards, HRP-labeled secondary antibody (1:5,000, Abcam) was reacted with the membrane which was then developed by enhanced chemiluminescence. GAPDH referred to an internal control. Target protein expression was calculated by gray analysis software.

\subsection{Statistical analysis}

Data were assessed with SPSS 21.0 (IBM, NY, USA) and measurement data were expressed as mean \pm standard deviation. Measurement data in normal distribution were compared by $t$ test between two groups, one-way analysis of variance (ANOVA) among multiple groups and Tukey's multiple comparisons test after ANOVA analysis. At $P<0.05$, statistical significance was established.

\section{Results}

\subsection{Induction of radioresistance in EC cells}

Colony formation assay was applied to assess the radioresistance of parental and radioresistant EC cells. The outcomes indicated that after irradiation at $0,2,4,6,8$ and $10 \mathrm{~Gy}$, respectively for 12 days, the number of formed colonies was decreased with the increase of the irradiation dose. Also, if irradiated at the same dose, increased number of colonies was formed by radioresistant EC cells rather than parental EC cells. Since the results indicated that radioresistant EC cells had stronger radioresistance and colonyforming ability, it was confirmed that radioresistant EC cells TE-1R and KYSE-150R were successfully established (Fig. 1A-D).

\subsection{Anlotinib inhibits proliferation, angiogenesis, migration and invasion of radioresistant EC cells}

Anlotinib has anti-tumor activity against various solid tumors, however, its effect on the anti-cancer effect of radiotherapy on EC is not yet clearly understood. To further explore this issue, we established the radioresistant EC cells and treated TE-1R and KYSE-150R cells with different concentrations of Anlotinib $(2,4$, and $8 \mu \mathrm{mol} / \mathrm{L})$. It was found from MTT assay that after Anlotinib treatment, the proliferation of TE$1 \mathrm{R}$ and KYSE-150R cells was impaired in a concentration-dependent manner (Fig. 2A). The inhibitory effect of Anlotinib on proliferation was more effective at $4 \mu \mathrm{mol} / \mathrm{L}$, therefore, Anlotinib at $4 \mu \mathrm{mol} / \mathrm{L}$ was used for later experiment.

In tube formation and Transwell assays, along with Western blot assay, we disclosed that after Anlotinib treatment, tumor angiogenesis, migration and invasion of TE-1R and KYSE-150R cells were inhibited, and protein expression of angiogenesis-related factors VEGF and bFGF was reduced (Fig. 2B-E).

\subsection{EphA2 expression is raised in radioresistant EC cells}

Next, we applied RT-qPCR and Western blot to measure EphA2 expression in vitro. The outcome reflected that EphA2 expression was higher in TE-1 and KYSE-150 cells than THEECs, and higher in TE-1R and 
KYSE-150R cells than TE-1 and KYSE-150 cells (Fig. 3A, B). In addition, we also found the up-regulated EphA2 in EC in the Starbase website (Fig. 3C).

\subsection{Inhibiting EphA2 enhances Anlotinib-mediated effects on radioresistant EC cells}

We utilized RT-qPCR and Western blot to test EphA2 expression in TE-1R and KYSE-150R cells, and revealed that Anlotinib treatment reduced EphA2 expression (Fig. 4A). Then, we applied siRNA-EphA2 or OE-EphA2 to down-regulate or up-regulate EphA2 in TE-1R and KYSE-150R cells, and treated cells with Anlotinib at $4 \mu \mathrm{mol} / \mathrm{L}$. Subsequently, we collected the data from in vitro cell function experiments which indicated that OE-EphA2-mediated up-regulation of EphA2 reversed the inhibitory effect of Anlotinib on VEGF and bFGF protein expression, as well as on proliferation, tumor angiogenesis and migration and invasion of TE-1R and KYSE-150R cells. By contrast, siRNA-EphA2-induced down-regulation of EphA2 further enhanced Anlotinib-mediated effects on TE-1R and KYSE-150R cells (Fig. 4B-F).

\subsection{Down-regulating EphA2 depresses proliferation, angiogenesis, migration and invasion of radioresistant EC cells}

Next, we further explored the effect of EphA2 on cells separately and transfected siRNA-EphA2 or OEEphA2 into TE-1R and KYSE-150R cells. At first, RT-qPCR and Western blot were employed to verify that EphA2 expression in cells was successfully down-regulated or up-regulated (Fig. 5A). Next, through in vitro cell function experiments, we noticed that silencing EphA2 reduced proliferation, tumor angiogenesis, migration and invasion, as well as VEGF and bFGF protein expression in cells, while restoring EphA2 had the opposite effects (Fig. 5B-F).

\subsection{Anlotinib suppresses growth and angiogenesis of radioresistant EC cells in vivo}

The tumor formation rate of 24 nude mice was $100 \%$, and no natural death occurred during the experiment. For mice exposed to irradiation, it was recognized that tumor volume, weight and EphA2 expression were all suppressed. Then, further treatment of Anlotinib was found to enhance the inhibitory effects of irradiation on mice (Fig. 6A-E).

\section{Discussion}

Radiation has an established role in definitive, palliative and neoadjuvant environments, having a vital effect on the treatment of local EC [19]. To date, multiple drugs have been introduced to overcome radioresistance in EC, including Anlotinib. In our research, we have recognized the therapeutic efficacy of Anlotinib and further disclosed the underlying mechanism of Anlotinib by regulating EphA2 in EC.

Collectively, Anlotinib could induces tumor blood vessel normalization to strengthen the anticancer effect of radiotherapy in EC by inhibiting EphA2. 
To specify the action of Anlotinib in redioresistance of EC, we administrated Anlotinib at $4 \mu \mathrm{mol} / \mathrm{L}$ to treat radioresistant EC cells and observed its inhibitory impacts on cellular proliferation, angiogenesis, migration and invasion, as well as tumor growth. In a case report, it has been observed that administration of Anlotinib has a better response for the fourth-line therapy and prolongs the overall survival time of patients with ESCC [11]. In another clinical trail, it has been noticed that combined administration of nivolumab and Anlotinib as a second-line therapy could improve the physical condition of the patient with advanced ESCC [20]. In addition to that, a recent report has highlighted that anlotinib and chemoradiotherapy in combination has the ideal an-tumor effect to treat ESCC in mice [10]. Not only limited to EC, treatment of Anlotinib works actively in other cancer types. For instance, treatment of Anlotinib in thyroid cancer cells causes impairments in cell viability and migration in vitro and tumor growth in vivo [21]. Moreover, some studies have emerged the regulatory mechanism of Anlotinib in suppressing tumorigenesis. As exampled by a late paper, it is revealed that Anlotinib could limit lung cancer cells to proliferate, invade and migrate and tumors to grow aggressively by blocking the mitogenactivated protein kinase/extracellular signal-regulated kinase (ERK) pathway [22]. Other than that, Anlotinib-induced inhibition on proliferation, migration, invasion and tube formation, as well as tumorigenicity in vivo is recognized in colorectal cancer through suppressing AKT/ERK pathway [18]. Our study also mentioned that Anlotinib also exhibited great effects on promoting the efficacy of radiotherapy in EC. Consistently, it is noted that in the setting of lung cancer, the synergism of radiotherapy and Anlotinib is more effective to suppress cell proliferation and tumor cell growth than administration of Anlotinib alone [23]. Overall, Anlotinib is a promising drug for managing the process of cancer and improving the survival of cancer patients; moreover, Anlotinib and radiotherapy synergistically function to control tumorigenic activities of malignant cells.

Next, we studied the role and actions of EphA2 in EC, and found it being up-regulated EC cell lines and radioresistant EC cells. Subsequently, we performed cell function assays and finally uncovered that up/down-regulating EphA2 enhanced/impaired the proliferation, invasion, migration and angiogenesis of redioresistant EC cells. Deeply, we analyzed the synergism of EphA2 and Anlotinib, and revealed that inhibiting EphA2 strengthened the effects of Anlotinib on redioresistant EC cells. In fact, a phosphotyrosine profiling has indicated that EphA2 expression is raised in ESCC, and knocking down EphA2 could decrease proliferation and invasion of malignant cells [24]. Other researches have also identified the role of EphA2 in various tumors. For examples, EphA2 expression is elevated in small-cell lung cancer, and suppression of EphA2 has the ability to restrain cell proliferation [25]. Concerning to the regulatory role of EphA2 in cancer radioresistance, it has been described that blocking EphA2 could suppress radioresistance of NSCLC cells, as well as the migration, proliferation, and invasion of malignant cells [26]. It is known that miR-200c-induced radiosensitivity, as well as invasion, migration and tube formation reduction, is associated to EphA2 down-regulation in human cancer cells [27]. Anyway, targeted-down-regulation of EphA2 is an effective approach to induce radiosensitivity of EC cells.

\section{Conclusion}


In summary, our research provides a novel perspective to the regulatory mechanism of Anlotinib in EC, and delineates that Anlotinib could inhibit EphA2 expression, thus to suppress angiogenesis and resensitize EC cells to radiotherapy. However, our study is in preclinical level, and much efforts are required to develop the results in clinics.

\section{List Of Abbreviation}

EC esophageal cancer

EphA2 Ephrin type-A receptor 2

ESCC esophageal squamous cell carcinoma

MVD microvessel density

NSCLC non-small cell lung cancer

THEECs Human normal esophageal epithelial cells

RPMI Roswell Park Memorial Institute

FBS fetal bovine serum

OE overexpression

$\mathrm{CM}$ conditioned medium

DMEM Dulbecco's Modified Eagle Medium

RT-qPCR Reverse transcription quantitative polymerase chain reaction

GAPDH glyceraldehyde-3-phosphate dehydrogenase

ANOVA analysis of variance

\section{Declaration}

\section{Funding}

The study is funded by Huai 'an Natural Science Research Program (No.HAB202026); Science Development Foundation of Nanjing Medical University (No.NMUB2020153)

\section{Conflict of interest}

The authors declare that they have no conflicts of interest.

\section{Ethical statement}


Animal experiments have been reviewed and approved by the animal ethics committee of The Affiliated Huaian No.1 People's Hospital of Nanjing Medical University.

\section{Consent for publication}

Not applicable

\section{Availability of data and material}

Not applicable

\section{Authors' contributions}

Weiguo Zhu finished study design, Jing Huang, Jiasheng Huang, Yingying Xu finished experimental studies, Zhenlin Gu, Wanwei Wang finished data analysis, Jing Huang, Zhenlin Gu finished manuscript editing. All authors read and approved the final manuscript.

\section{Acknowledgement}

We would like to acknowledge the reviewers for their helpful comments on this paper.

\section{References}

1. Krug S, Michl PE, Cancer (2017) New Insights into a Heterogenous Disease. Digestion 95(4):253-261

2. Huang FL, Yu SJ (2018) Esophageal cancer: Risk factors, genetic association, and treatment. Asian J Surg 41(3):210-215

3. Short MW, Burgers KG, Fry VTE (2017) Cancer Am Fam Physician 95(1):22-28

4. Kato H, Nakajima M (2013) Treatments for esophageal cancer: a review. Gen Thorac Cardiovasc Surg 61(6):330-335

5. Chen GZ, Zhu HC, Dai WS, Zeng XN, Luo JH, Sun XC (2017) The mechanisms of radioresistance in esophageal squamous cell carcinoma and current strategies in radiosensitivity. J Thorac Dis 9(3):849-859

6. Arjaans M, Oude Munnink TH, Oosting SF, Terwisscha van Scheltinga AG, Gietema JA, Garbacik ET et al (2013) Bevacizumab-induced normalization of blood vessels in tumors hampers antibody uptake. Cancer Res 73(11):3347-3355

7. Syed YY, Anlotinib (2018) First Global Approval. Drugs 78(10):1057-1062

8. Lin B, Song X, Yang D, Bai D, Yao Y, Lu N (2018) Anlotinib inhibits angiogenesis via suppressing the activation of VEGFR2, PDGFRbeta and FGFR1. Gene 654:77-86

9. Shen G, Zheng F, Ren D, Du F, Dong Q, Wang Z et al (2018) Anlotinib: a novel multi-targeting tyrosine kinase inhibitor in clinical development. J Hematol Oncol 11(1):120

10. Shi J, Zhang Y, Wang J, Li J, Li ZA (2020) Combined With Chemoradiotherapy Exhibits Significant Therapeutic Efficacy in Esophageal Squamous Cell Carcinoma. Front Oncol 10:995

11. Yang D, Xu F, Lai X, Li Y, Gao H, Xu Y et al (2020) Combined treatment with anlotinib and chemotherapy for advanced esophageal squamous cell carcinoma improved patient survival: a case report. Am J Transl Res 12(10):6578-6583 
12. Xiao T, Xiao Y, Wang W, Tang YY, Xiao Z, Su M (2020) Targeting EphA2 in cancer. J Hematol Oncol 13(1):114

13. Shi H, Yu F, Mao Y, Ju Q, Wu Y, Bai W et al (2018) EphA2 chimeric antigen receptor-modified T cells for the immunotherapy of esophageal squamous cell carcinoma. J Thorac Dis 10(5):2779-2788

14. Zhang J, Deng G, Qiao L, Luo H, Liu Q, Liang N et al (2018) Effect of galectin-3 on vasculogenic mimicry in esophageal cancer cells. Oncol Lett 15(4):4907-4911

15. Weng G, Zhao W, Yin Y, Wang S, Du L, Liu N et al (2020) Genomic alterations of whole exome sequencing in esophageal squamous cell carcinoma before and after radiotherapy. $\mathrm{J}$ Thorac Dis 12(10):5945-5957

16. Merritt WM, Kamat AA, Hwang JY, Bottsford-Miller J, Lu C, Lin YG et al (2010) Clinical and biological impact of EphA2 overexpression and angiogenesis in endometrial cancer. Cancer Biol Ther 10(12):1306-1314

17. Jing Z, Gong L, Xie CY, Zhang L, Su HF, Deng X et al (2009) Reverse resistance to radiation in KYSE150R esophageal carcinoma cell after epidermal growth factor receptor signal pathway inhibition by cetuximab. Radiother Oncol 93(3):468-473

18. Yang Q, Ni L, Imani S, Xiang Z, Hai R, Ding R et al (2020) Anlotinib Suppresses Colorectal Cancer Proliferation and Angiogenesis via Inhibition of AKT/ERK Signaling Cascade. Cancer Manag Res 12:4937-4948

19. Zhang M, Wu (2017) AJ Radiation techniques for esophageal cancer. Chin Clin Oncol 6(5):45

20. Tang Y, Ou Z, Yao Z, Qiao (2019) G A case report of immune checkpoint inhibitor nivolumab combined with anti-angiogenesis agent anlotinib for advanced esophageal squamous cell carcinoma. Medicine 98(40):e17164

21. Ruan X, Shi X, Dong Q, Yu Y, Hou X, Song X et al (2019) Antitumor effects of anlotinib in thyroid cancer. Endocr Relat Cancer 26(1):153-164

22. Hu H, Liu Y, Tan S, Xie XX, He J, Luo F et al (2020) Anlotinib Exerts Anti-Cancer Effects on KRASMutated Lung Cancer Cell Through Suppressing the MEK/ERK Pathway. Cancer Manag Res 12:3579-3587

23. Guo L, Zhang L, Guan Y, Li Y, Zhang C, Guo (2021) Q In vitro studies of H520 cell cycle and apoptosis by anlotinib combined with radiotherapy. Thorac Cancer 12(5):593-602

24. Syed N, Barbhuiya MA, Pinto SM, Nirujogi RS, Renuse S, Datta KK et al (2015) Phosphotyrosine profiling identifies ephrin receptor $\mathrm{A} 2$ as a potential therapeutic target in esophageal squamous-cell carcinoma. Proteomics 15(2-3):374-382

25. Ishigaki H, Minami T, Morimura O, Kitai H, Horio D, Koda Y et al (2019) EphA2 inhibition suppresses proliferation of small-cell lung cancer cells through inducing cell cycle arrest. Biochem Biophys Res Commun 519(4):846-853

26. Gong S, Li Y, Lv L, Men W, Restored microRNA-519a enhances the radiosensitivity of non-small cell lung cancer via suppressing EphA2. Gene Ther. 2021 
27. Koo T, Cho BJ, Kim DH, Park JM, Choi EJ, Kim HH et al (2017) MicroRNA-200c increases radiosensitivity of human cancer cells with activated EGFR-associated signaling. Oncotarget 8(39):65457-65468

\section{Figures}

A
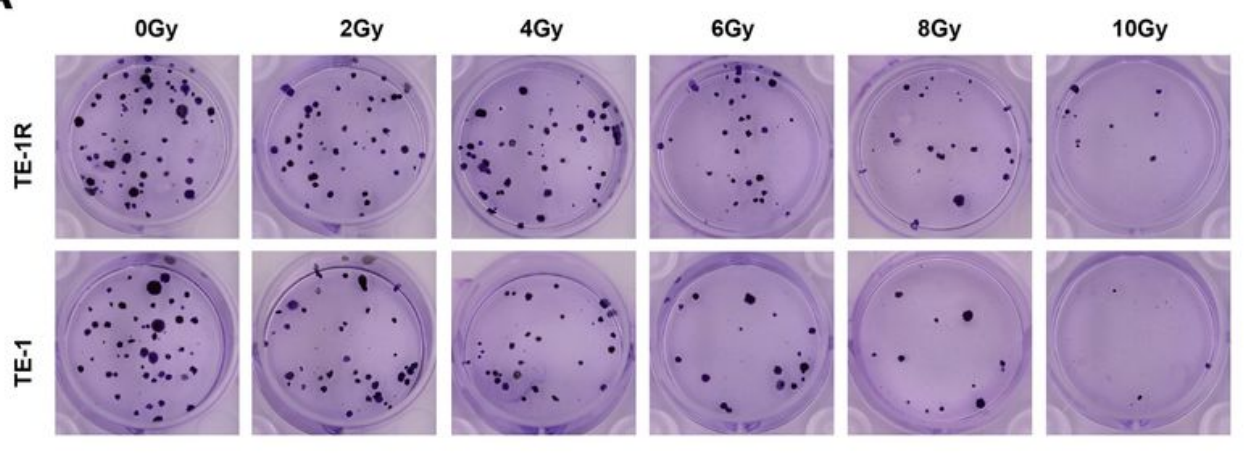

B

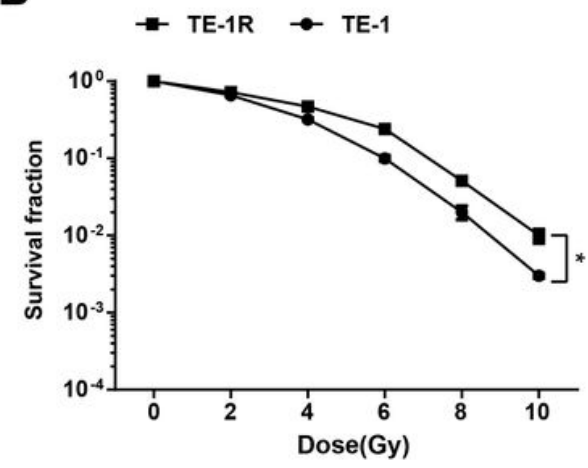

C

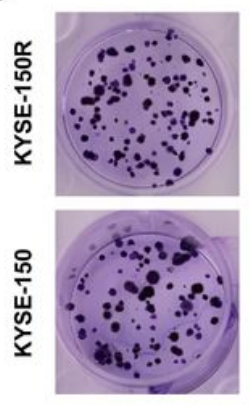

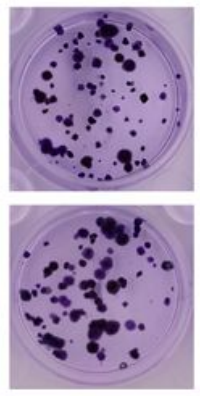

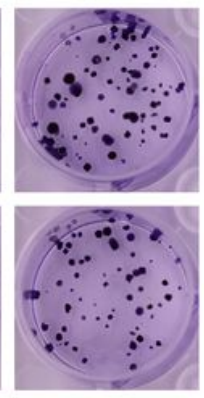

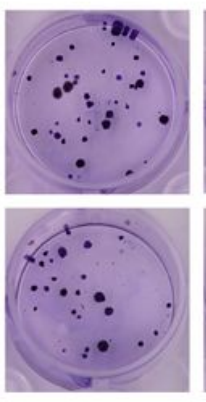
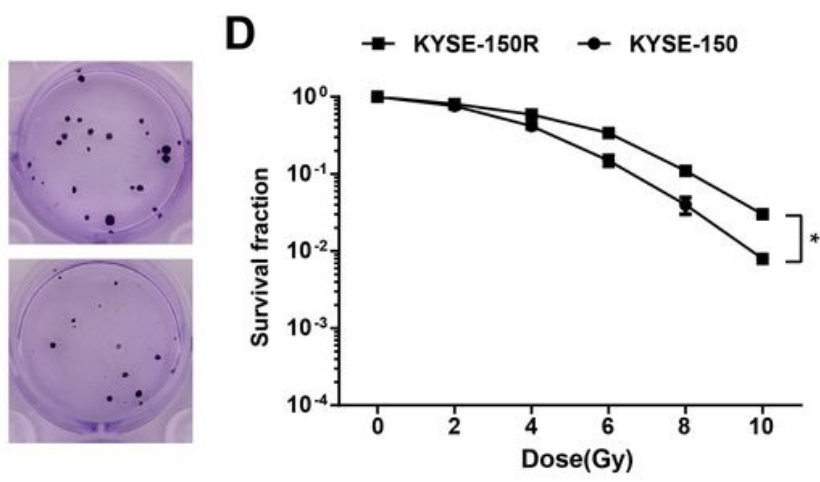

Figure 1

Induction of radioresistance in EC cells. A. Colony-forming ability of TE-1 and TE-1R cells irradiated with different doses. B. Survival curve of TE-1 and TE-1R cells irradiated with different doses. C. Colonyforming ability of KYSE-150 and KYSE-150R cells irradiated with different doses. D. Survival curve of KYSE-150 and KYSE-150R cells irradiated with different doses. ${ }^{*} P<0.05,{ }^{*} P<0.01$; Repetition $=3$; the data were expressed in the form of mean \pm standard deviation and compared by $t$ test. 
A

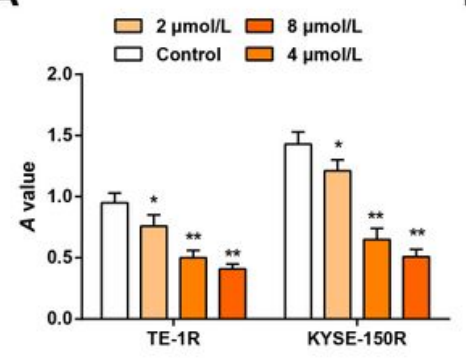

C

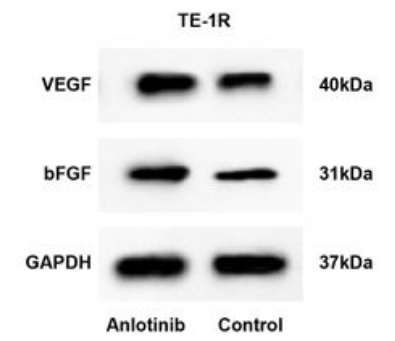

D

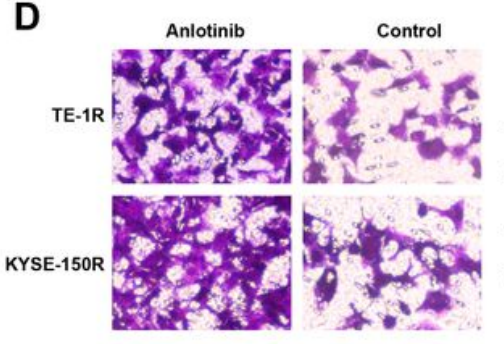

B
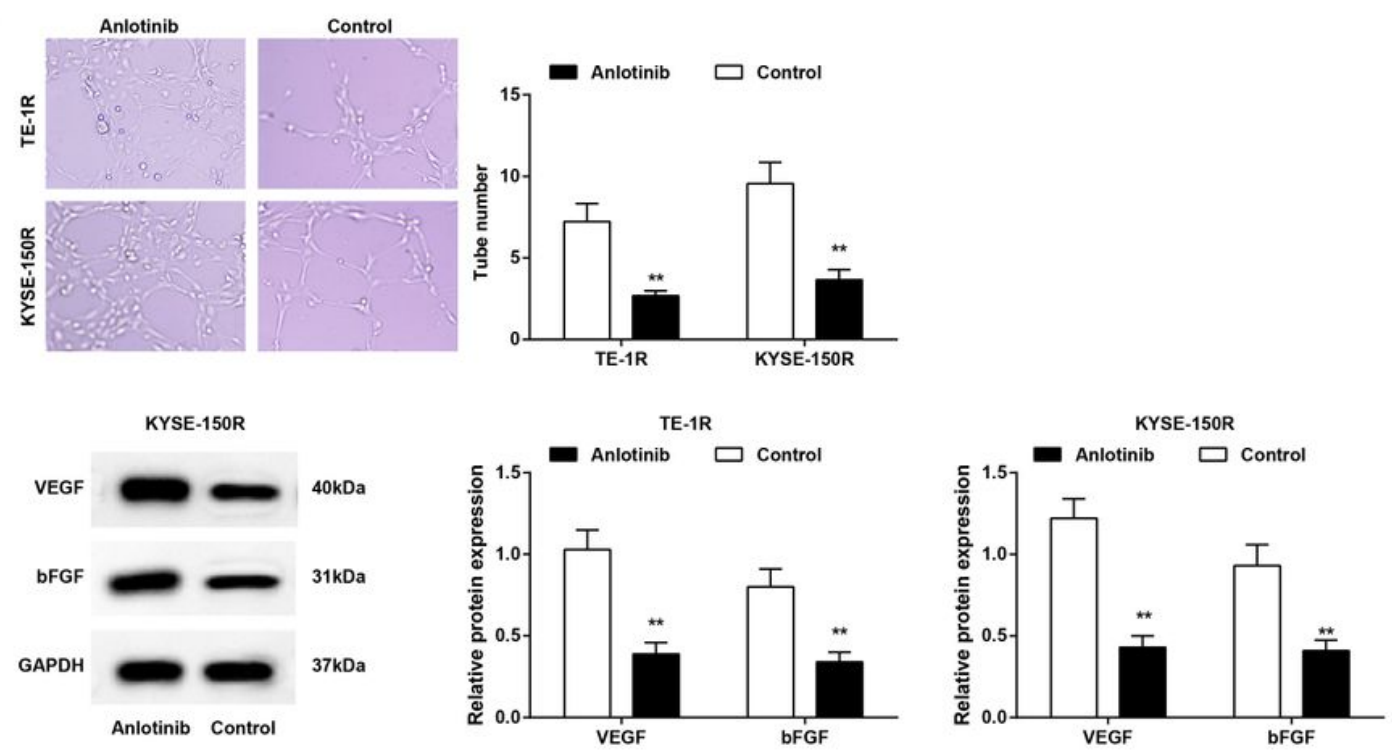

E

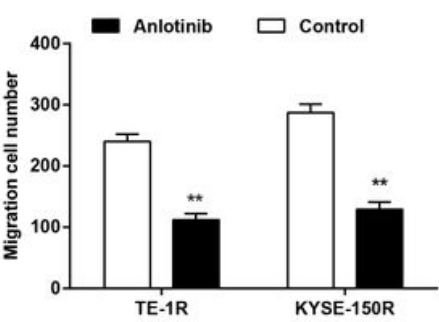

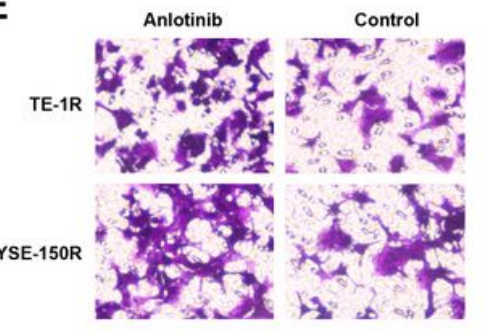

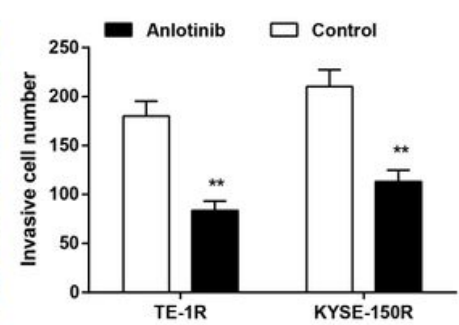

Figure 2

Anlotinib inhibits proliferation, angiogenesis, migration and invasion of radioresistant EC cells. A. MTT assay detected cell proliferation; $\mathrm{B}$. Tube formation assay detected cell angiogenesis; C. Western blot detected VEGF and bFGF expression in cells; D. Transwell assay detected cell migration; E. Transwell assay detected cell invasion; * $P<0.05$, $* \star P<0.01$; Repetition $=3$; the data were expressed in the form of mean \pm standard deviation. Data were compared by t test (two groups), or one-way ANOVA (multiple groups) and Tukey's multiple comparisons test (pairwise comparison).

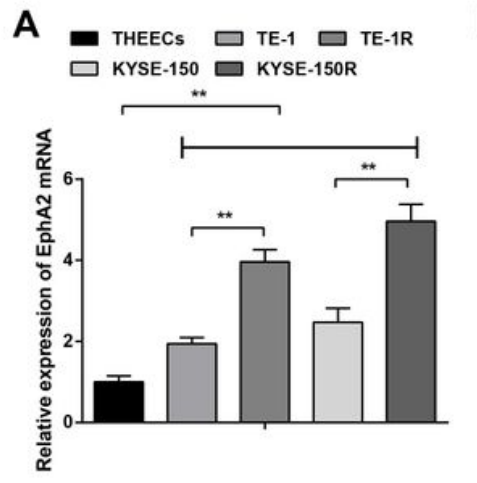

B

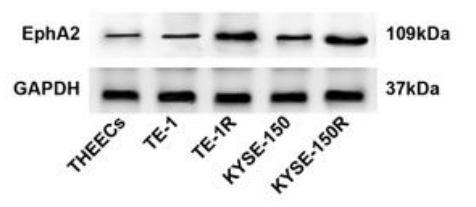

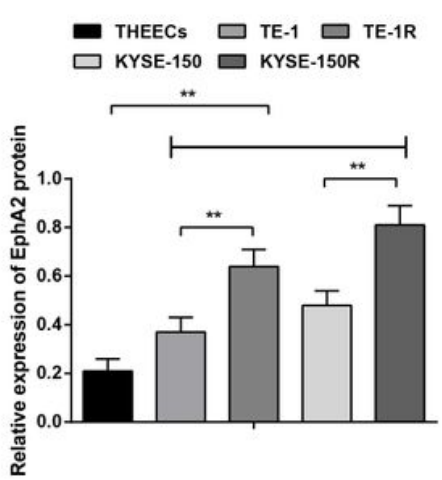

C

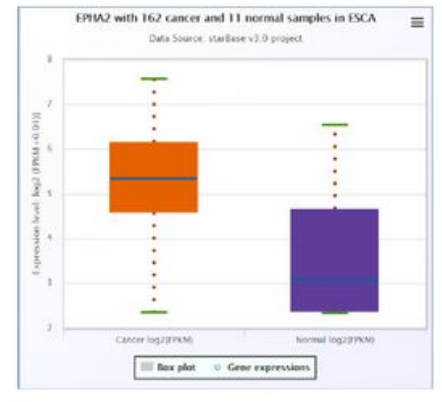

Figure 3 
EphA2 expression is raised in radioresistant EC cells. A. RT-qPCR detected EphA2 expression in THEECs, EC cell lines (TE-1 and KYSE-150) and radioresistant EC cells (TE-1R and KYSE-150R); B. Western blot detected EphA2 expression in THEECs, EC cell lines (TE-1 and KYSE-150) and radioresistant EC cells (TE$1 R$ and KYSE-150R); C. Starbase predicted that EphA2 was up-regulated in EC; * $P<0.05$, $*$ $P<0.01$; Repetition $=3$; the data were expressed in the form of mean \pm standard deviation and compared by oneway ANOVA and Tukey's multiple comparisons test.
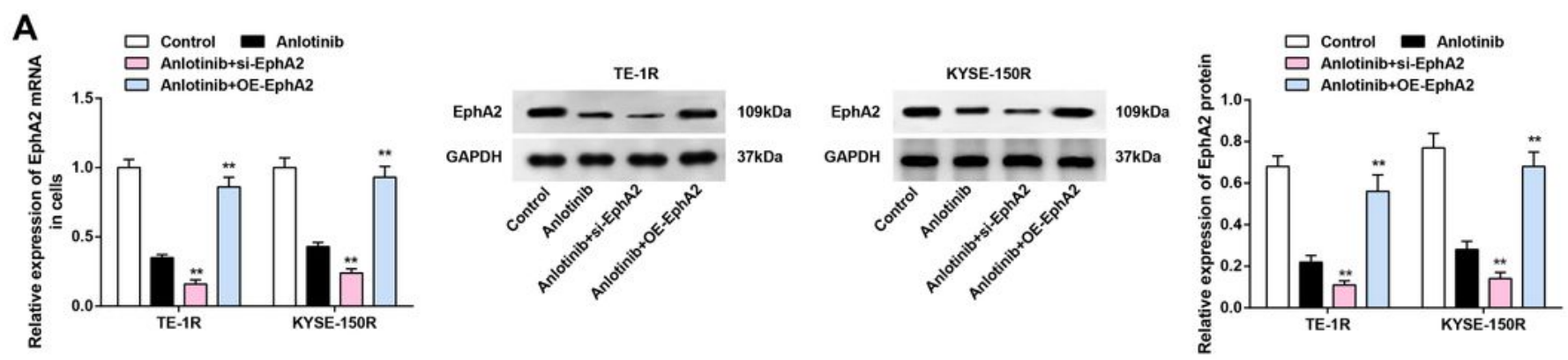

B

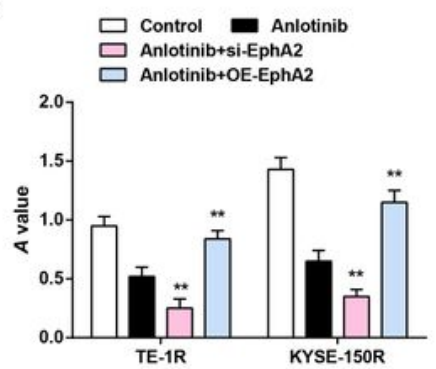

C
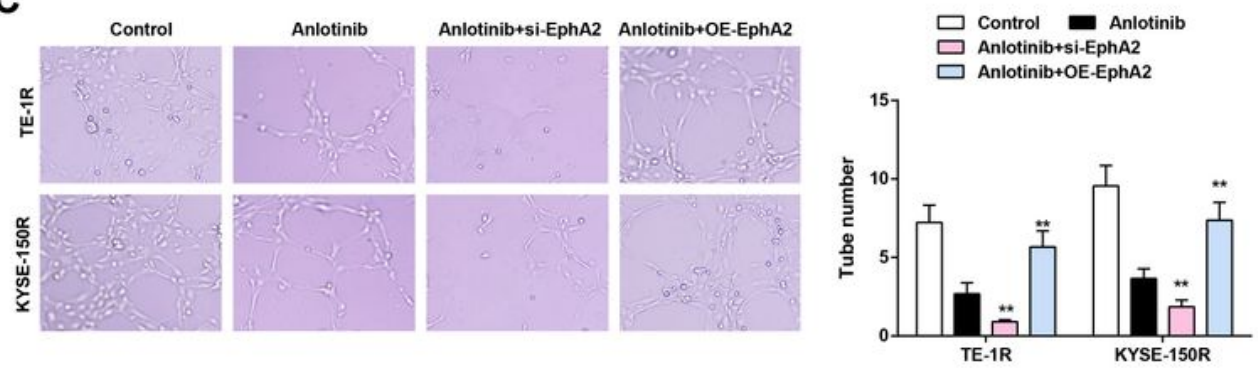

D

TE-1R

KYSE-150R

TE-1R
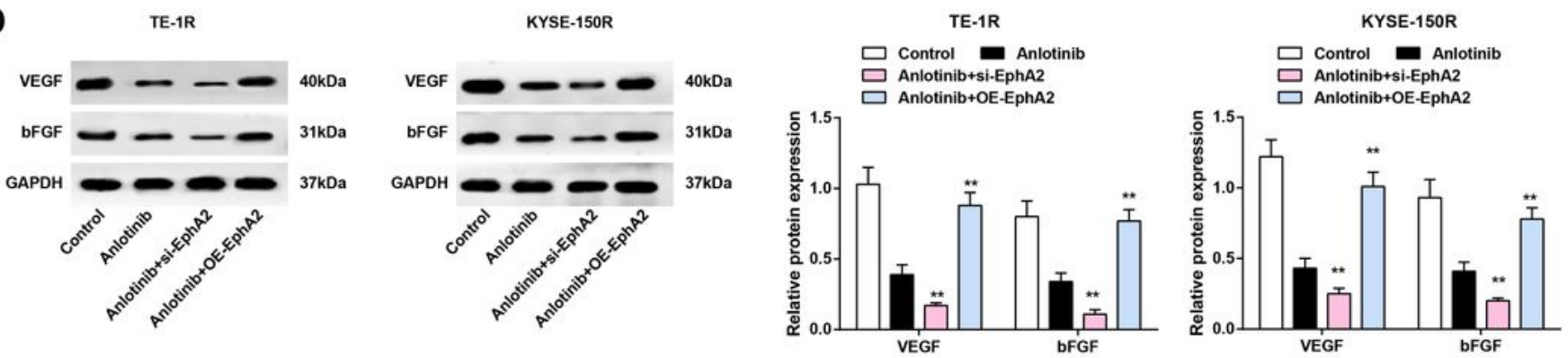

E
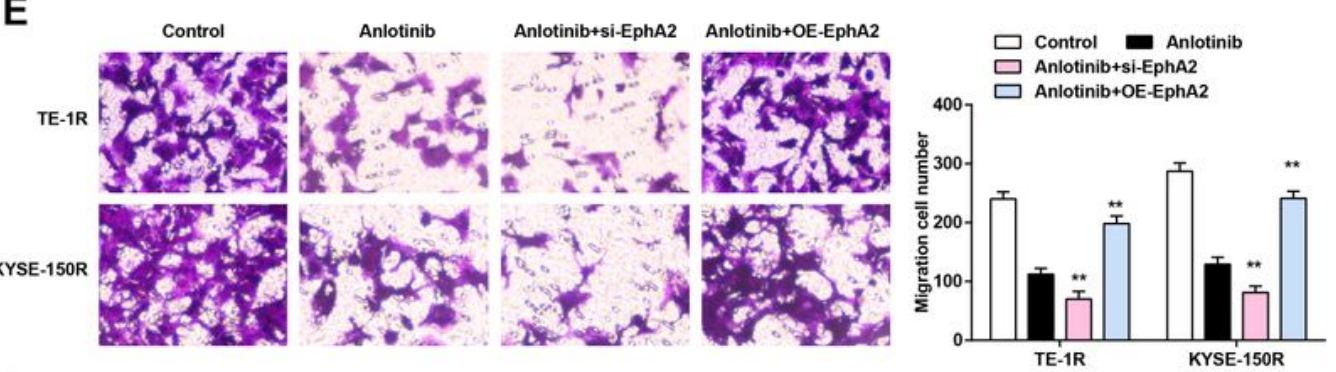

$\mathbf{F}$
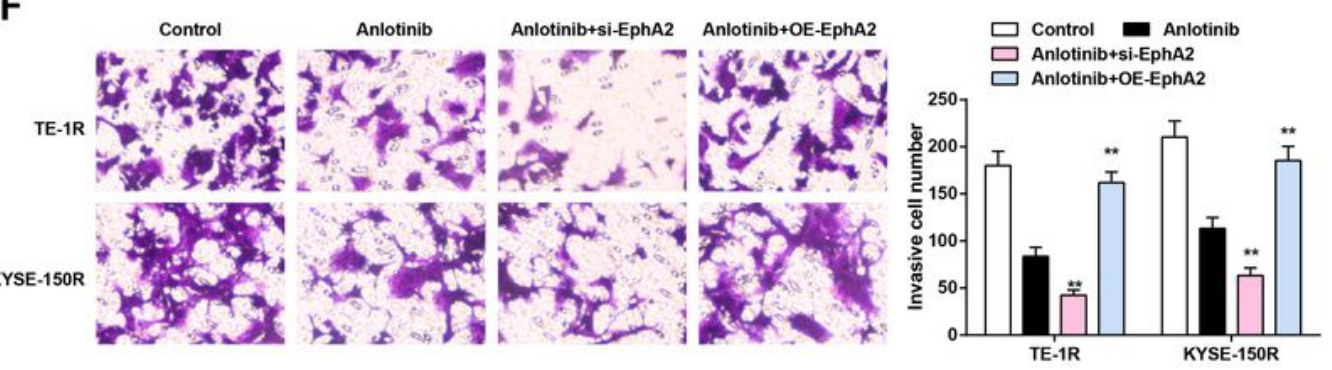

Figure 4 
Inhibiting EphA2 enhances Anlotinib-mediated effects on radioresistant EC cells. A. RT-qPCR and Western blot detected EphA2 expression in cells; B. MTT assay detected cell proliferation; C. Tube formation assay detected cell angiogenesis; D. Western blot detected VEGF and bFGF expression in cells; E. Transwell assay detected cell migration; $F$. Transwell assay detected cell invasion; $*<0.05, * * P<0.01$; Repetition $=3$; the data were expressed in the form of mean \pm standard deviation and compared by one-way ANOVA and Tukey's multiple comparisons test.
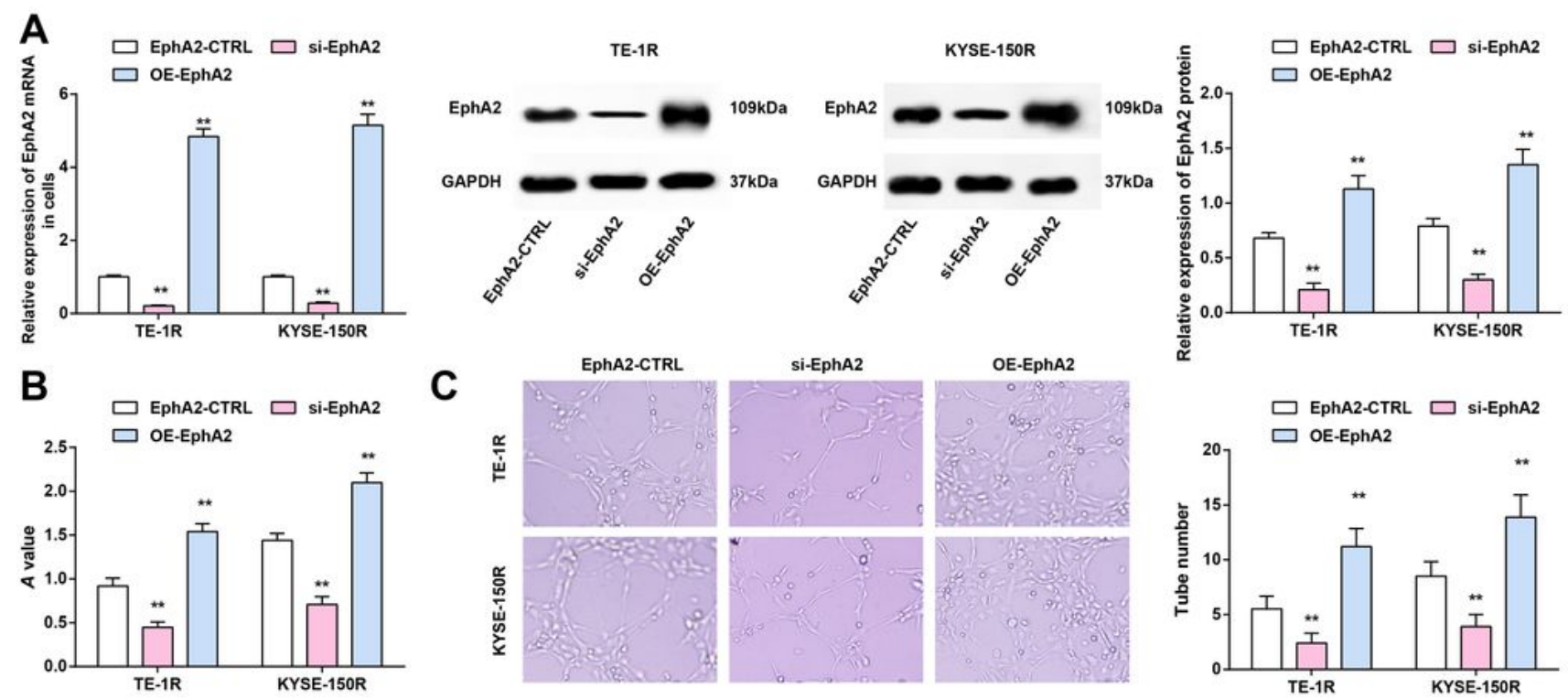

\section{D}
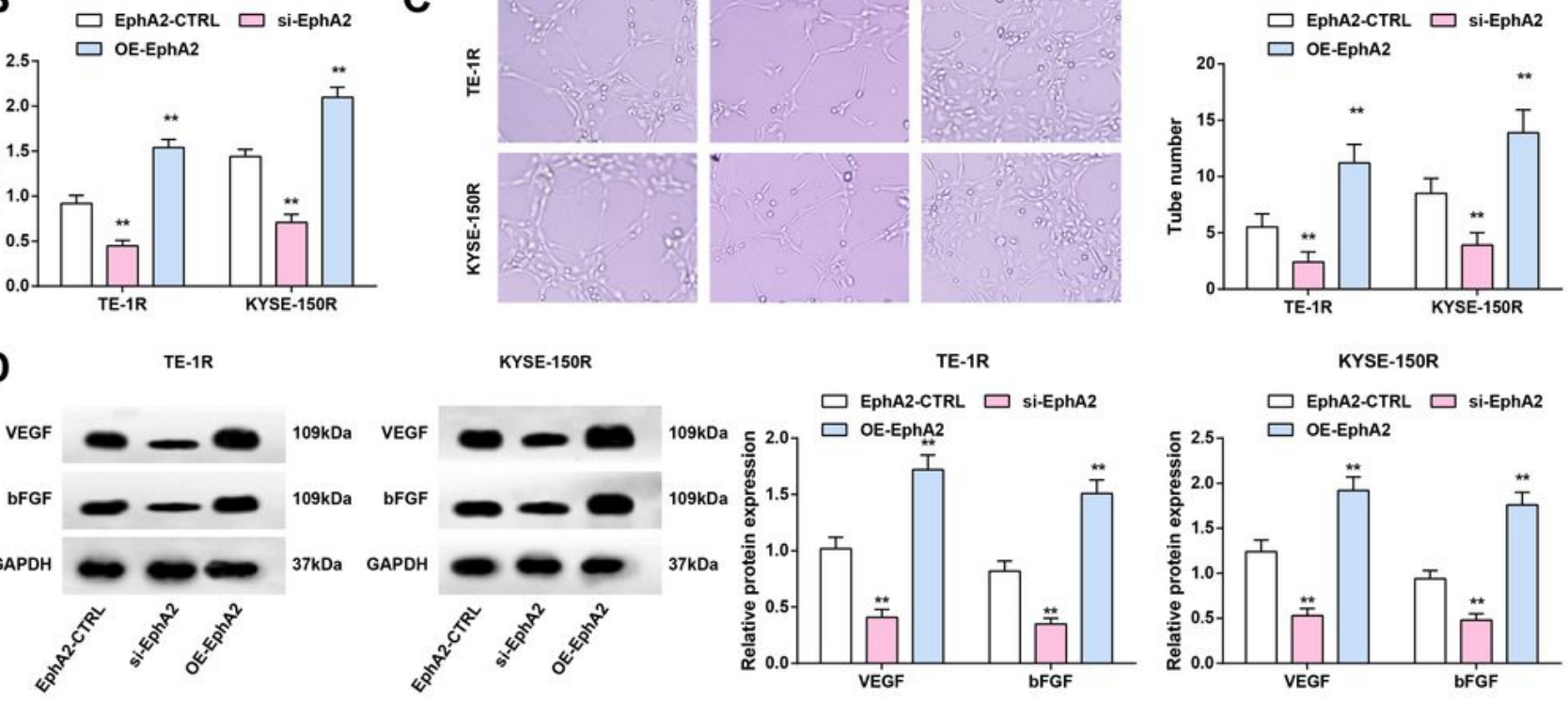

E
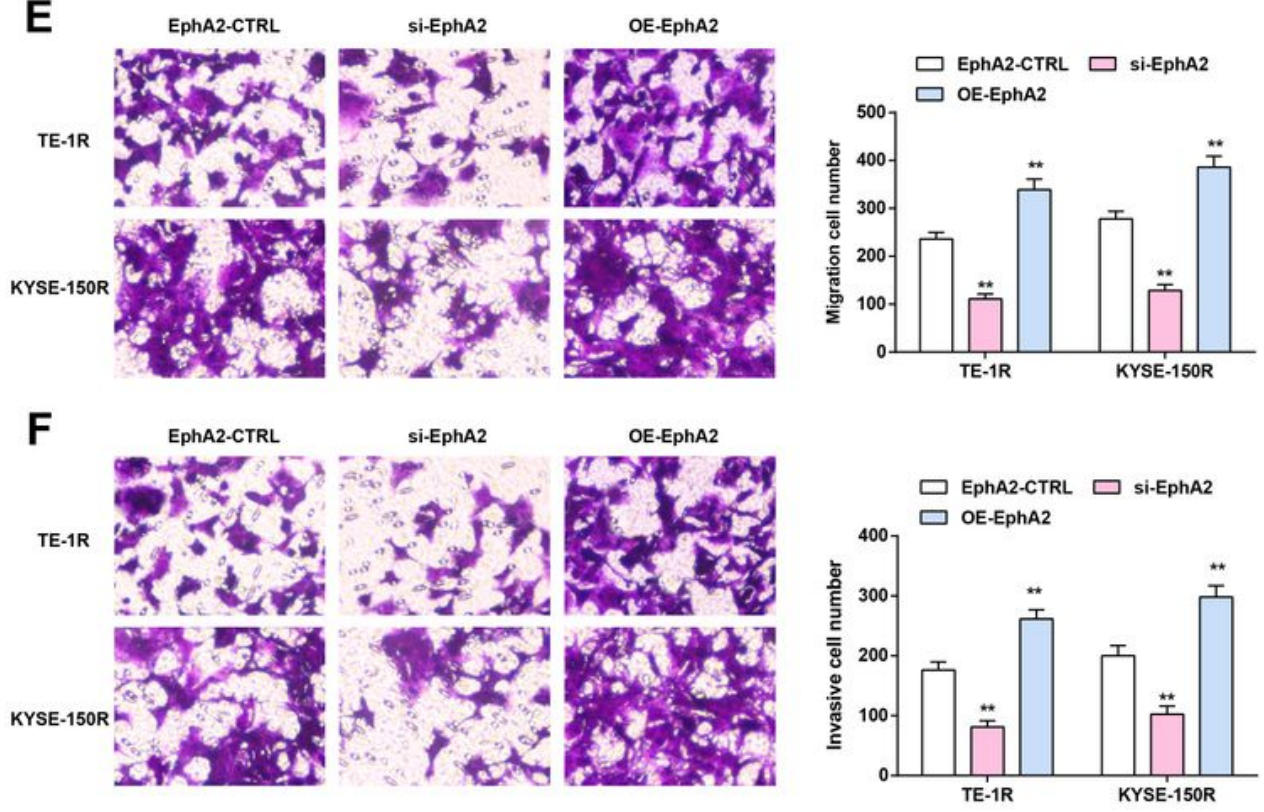

Figure 5 
Down-regulating EphA2 depresses proliferation, angiogenesis, migration and invasion of radioresistant EC cells. A. RT-qPCR and Western blot detected EphA2 expression in cells; B. MTT assay detected cell proliferation; C. Tube formation assay detected cell angiogenesis; D. Western blot detected VEGF and bFGF expression in cells; E. Transwell assay detected cell migration; F. Transwell assay detected cell invasion; $* P<0.05$, $* * P<0.01$; Repetition $=3$; the data were expressed in the form of mean \pm standard deviation and compared by one-way ANOVA and Tukey's multiple comparisons test.
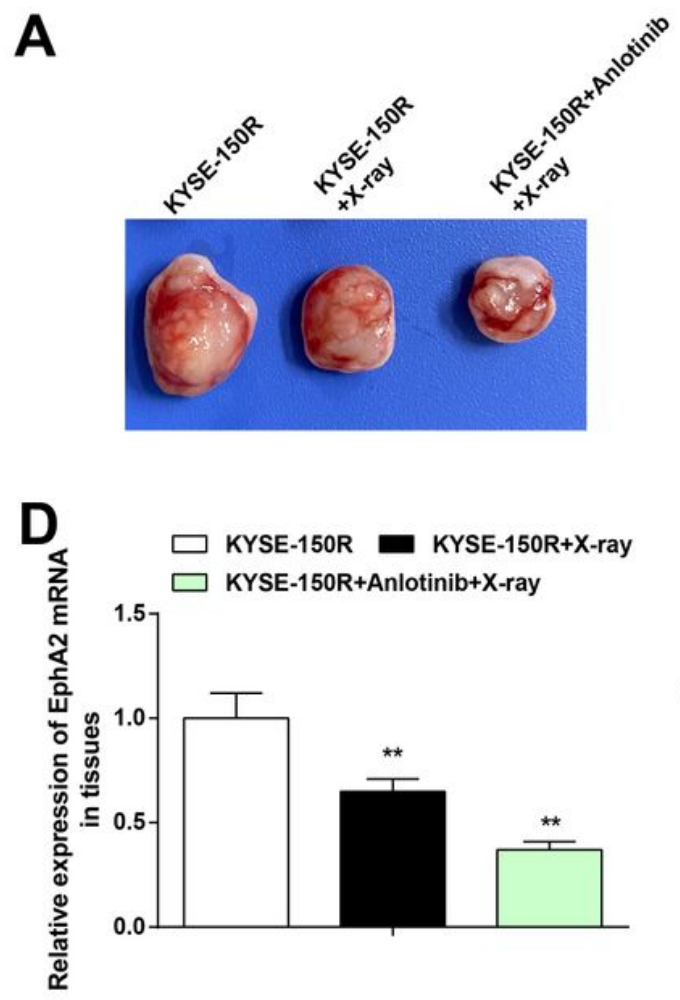
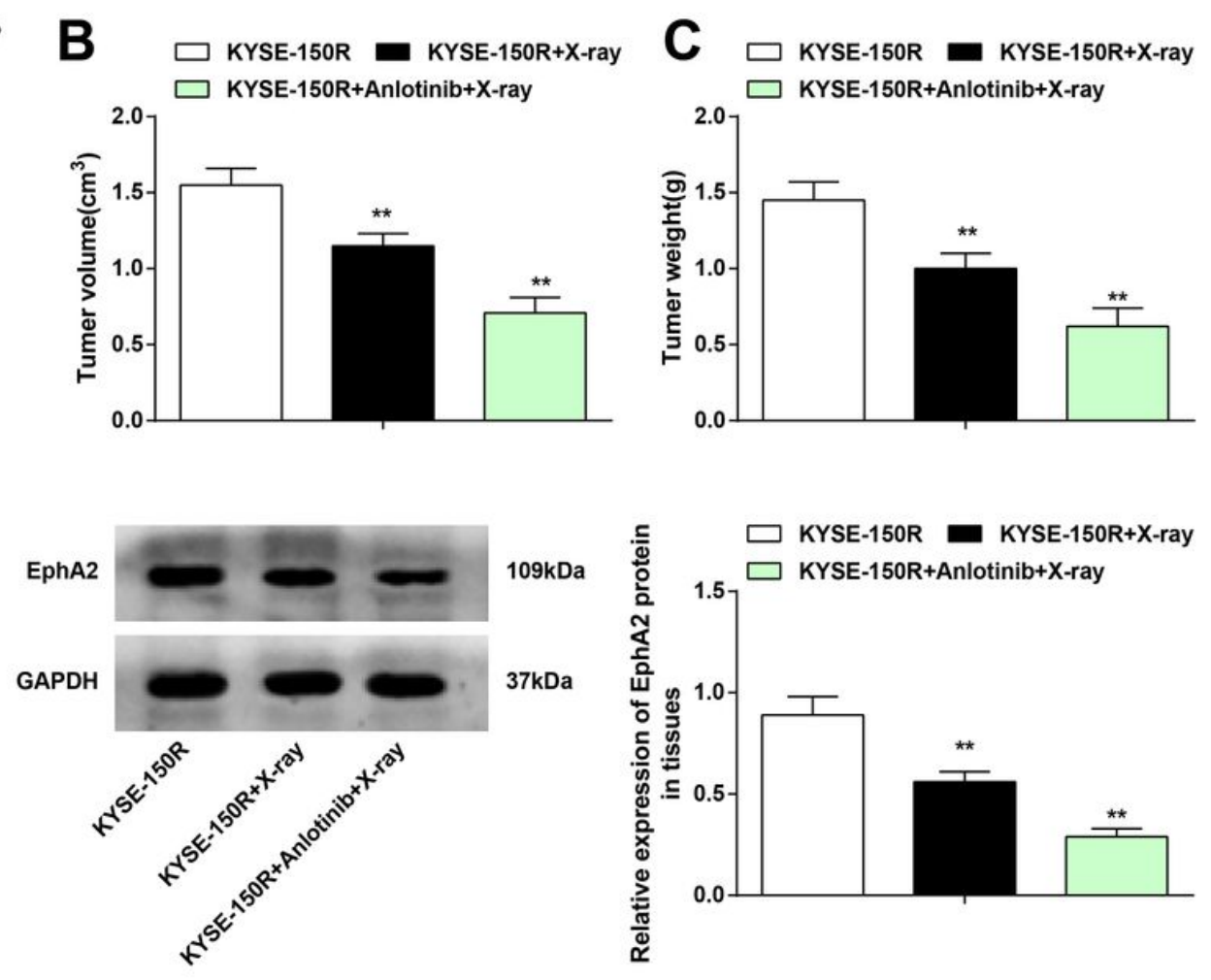

\section{Figure 6}

Anlotinib suppresses growth and angiogenesis of radioresistant EC cells in vivo. A. Representative images of tumors; B. Tumor volume in nude mice; $C$. Tumor weight in nude mice; D. RT-qPCR and Western blot detected EphA2 expression in tumors of nude mice; ${ }^{*} P<0.05$, ${ }^{*} P<0.01 ; n=5$; the data were expressed in the form of mean \pm standard deviation and compared by one-way ANOVA and Tukey's multiple comparisons test. 